\title{
Primary Appendiceal Adenocarcinoma Masquerading as Chronic Pelvic Pain
}

\author{
Douglas N. Brown, MD, Christina L. Jones, DO, Difu Wu, MD, Joseph M. Gobern, MD \\ Department of Obstetrics and Gynecology, Walter Reed National Military Medical Center Bethesda, Bethesda, MD, USA \\ (Drs. Brown, Jones, Gobern). \\ Department of Pathology, Walter Reed National Military Medical Center Bethesda, Bethesda, MD, USA (Dr. Wu).
}

\begin{abstract}
Introduction: Primary appendiceal adenocarcinoma is an exceedingly rare malignancy of the gastrointestinal tract that most often presents with a clinical picture consistent with acute appendicitis. These appendiceal tumors are frequently diagnosed intraoperatively and are almost never diagnosed preoperatively. In contrast, chronic pelvic pain is a commonly encountered clinical condition that has a plethora of possible etiologies that are often treated medically. In the atypical patient presentation, laparoscopy provides the ultimate diagnostic modality in addition to providing a viable therapeutic surgical option.

Case Description: We present a case of primary appendiceal adenocarcinoma masquerading as chronic pelvic pain in a 32-year-old female patient and discuss the importance of diagnostic laparoscopy in the diagnosis and management of this unusual clinical scenario.

Discussion: Though rare in the young female patient, the possibility of malignancy must always be entertained within the differential diagnosis of chronic pelvic pain. When medical therapy for presumed chronic pelvic pain fails a 3- to 6-month empiric trial, the patient should undergo a full re-evaluation including diagnostic laparoscopy. It is important for the gynecologist and gynecologic oncologist to be familiar with the clinical and pathologic features in addition to the surgical management of these malignancies because primary appendiceal adenocarcinomas may masquerade as chronic pelvic pain.
\end{abstract}

Key Words: Appendiceal adenocarcinoma, Chronic pelvic pain, Laparoscopy.

Citation Brown DN, Jones CL, Wu D, Gobern JM. Primary appendiceal adenocarcinoma masquerading as chronic pelvic pain. CRSLS e2014.00109. DOI 10.4293/CRSLS.2014.00109

Copyright (c) 2014 SLS This is an open-access article distributed under the terms of the Creative Commons Attribution-Noncommercial-ShareAlike 3.0 Unported license, which permits unrestricted noncommercial use, distribution, and reproduction in any medium, provided the original author and source are credited.

Address correspondence to: Douglas N. Brown, MD, Department of Obstetrics and Gynecology, Walter Reed National Military Medical Center Bethesda, 8901 Wisconsin Ave, Bethesda, MD 20889-5600, USA. Telephone: (301) 400-2686, Fax: (301) 295-0764, E-mail: dnbrownsu@gmail.com

\section{INTRODUCTION}

Primary appendiceal adenocarcinoma is an infrequently encountered malignancy representing $<0.5 \%$ of all intestinal neoplasms. ${ }^{1}$ These tumors are not often associated with distinct clinical manifestations and are usually discovered incidentally at the time of appendectomy for suspected appendicitis. Mucinous adenocarcinoma is the most frequently encountered histology, followed by colonic type, goblet cell carcinoid, malignant carcinoid, and signet ring cell. ${ }^{2}$ The role of laparoscopy in the surgical management of these neoplasms is becoming more pro- nounced within the literature, specifically in those patients with low-grade tumor histology and no evidence of cecal involvement. 3,4 Whereas primary appendiceal adenocarcinoma is uncommon in young female patients, with a mean age of 49 years at the time of diagnosis, chronic pelvic pain is a frequently encountered clinical condition in this patient population. ${ }^{5}$ The possibility of malignancy must always be considered within the differential diagnosis of chronic pelvic pain, even in the youngest of patients. We report a case of a primary appendiceal adenocarcinoma masquerading as chronic pelvic pain in a young female patient and discuss the role of 


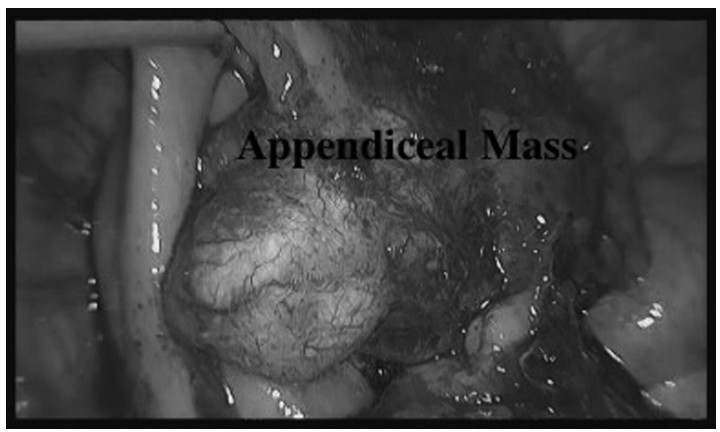

Figure 1. Appendiceal mass.

laparoscopy in the diagnosis and management of this unique clinical scenario.

\section{CASE REPORT}

The patient is a 32-year-old woman who presented to the Walter Reed National Military Medical Center (WRNMMC) Gynecology Clinic as a referral for a 2-year history of chronic pelvic pain that had been refractory to medical management at her overseas duty station. The patient's medical history was significant for a diagnosis of pelvic inflammatory disease (PID) due to a remote history of Chlamydia trachomatis. The original physical examination at her overseas duty station was notable for right adnexal fullness and tenderness to palpation. Endovaginal ultrasonography was performed at that time and showed evidence of a simple-appearing 3- to 4-cm right ovarian cyst, with a normal-appearing left ovary and normal uterus. No cervical motion tenderness was noted on the examination. At her overseas duty station, she was diagnosed with recurrent PID and a simple right ovarian cyst. She was initially prescribed antibiotic therapy, an estrogen-progestin oral contraceptive, and nonsteroidal antiinflammatory agents for pain. Over the course of the next 12 months, the patient was seen and treated by multiple providers and was prescribed various formulations of estrogen-progestin contraceptives and nonsteroidal anti-inflammatory agents without any resolution of her symptoms. At her 12-month follow-up visit, she was diagnosed with chronic pelvic pain and prescribed a 6-month trial of a gonadotropin-releasing hormone agonist. At her 18month follow-up visit overseas, the patient's symptoms had not resolved, and she requested a referral to the Gynecology Clinic at WRNMMC.

The patient presented to the Gynecology Clinic at WRNMMC with complaints of persistent, sharp, right-sided pelvic pain that was unresponsive to over-the-counter pain medications. Physical examination showed the patient to be afebrile and normotensive, with a normal heart rate, right lower quadrant tenderness to palpation but no rebound or guarding, and no McBurney sign. Pelvic examination showed an 8-week-size uterus with normal contour and mobility, no cervical motion tenderness, and no left adnexal masses or fullness but evidence of right adnexal fullness, a palpable 4- to 5-cm mass, and severe tenderness to palpation. Endovaginal ultrasonography was performed and showed a 3- to 4-cm anechoic cyst with a single internal septation within the right ovary and an indeterminate $4-\mathrm{cm}$ heterogeneous area of complex fluid adjacent to the right ovary. Considering the patient's age and medical history, the differential diagnosis included a developing tubo-ovarian abscess in the setting of PID, hydrosalpinx, endometrioma, large subacute hemorrhagic cyst, complex adnexal cyst, and ovarian malignancy. The following tumor markers were obtained: Carcinoembryonic Antigen (CEA), $3.2 \mathrm{mg} / \mathrm{mL}$; Carbohydrate Antigen 125 (CA125), $31 \mathrm{U} / \mathrm{mL}$; and Carbohydrate Antigen 19-9 (CA 19-90), $21 \mathrm{U} / \mathrm{mL}$. All markers were within normal limits. The patient was counseled, and informed consent was obtained for a diagnostic laparoscopy.

On entry into the abdomen, pseudomyxoma peritonei was noted throughout the pelvic and abdominal cavity. There was evidence of a right inguinal omental herniation and a right ovarian mass. Peritoneal washings were obtained, a right salpingo-oophorectomy was performed, and biopsy specimens were sent for frozen section. The pathologic analysis showed benign ovarian stroma consistent with an ovarian serous cystadenoma. Further dissection was performed at the base of the cecum, exposing a bulky appendiceal mass covered in deposits of mucin measuring approximately $4 \mathrm{~cm} \times 6 \mathrm{~cm}$ (Figure 1). An intraoperative consultation with the surgical oncology department was obtained, and per recommendations, multiple peritoneal, omental, and appendiceal biopsies were performed and the surgery was concluded. The final pathologic analysis showed low-grade appendiceal mucinous adenocarcinoma of the appendix (Figure $\mathbf{2 A}$ ). The mucinous tumor cells in the appendix were strongly CK20 positive, diffusely CDX2 positive, and diffusely CEA positive (Figure 2, B-D) but were negative for $\mathrm{CK} 7$ and estrogen receptor. This is an important characteristic that helps distinguish primary ovarian mucinous tumors from primary mucinous appendiceal tumors. ${ }^{6}$

The patient was counseled on the aforementioned findings, and approximately 3 weeks after her initial laparoscopy, she underwent optimal laparoscopic cytoreduction that included cecectomy with appendectomy, greater omentectomy, and resection of the falciform and round 


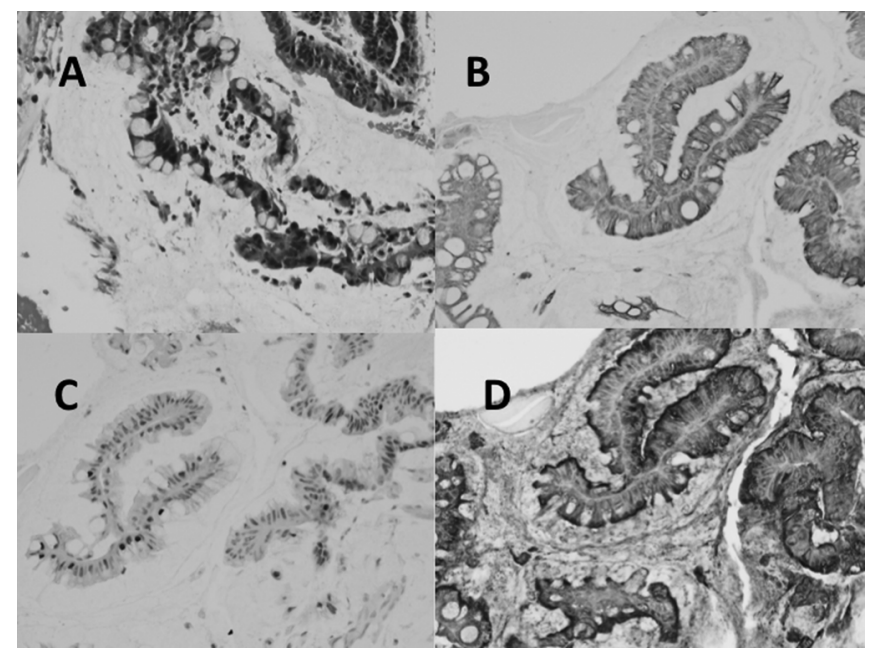

Figure 2. A, The mucinous tumor cells are columnar with atypical nuclei and an increased nuclear-to-cytoplasmic ratio (hematoxylin-eosin staining, original magnification $\times 200$ ). B, The mucinous tumor cells floating in pools of mucin in the appendix are strongly CK20 positive (CK20 staining, original magnification $\times 200$ ). C, The mucinous tumor cells floating in pools of mucin in the appendix are diffusely CDX2 positive (CDX2 staining, original magnification $\times 200$ ). D, The mucinous tumor cells floating in pools of mucin in the appendix are diffusely CEA positive (CEA staining, original magnification $\times 200)$.

ligament of the liver, followed by 90 minutes of heated intraperitoneal chemotherapy with mitomycin C. The patient's recovery went well, and her postoperative course was uneventful. Twenty-four months after her initial surgery, the patient is alive and well, without any evidence of disease.

\section{DISCUSSION}

Primary appendiceal adenocarcinoma is an exceedingly rare malignancy of the gastrointestinal tract, representing approximately 0.12 cases per 1000000 people per year. ${ }^{2}$ Fewer than $25 \%$ of these appendiceal neoplasms are diagnosed preoperatively. ${ }^{7}$ Most patients with mucinous appendiceal adenocarcinoma will present with a clinical picture consistent with acute appendicitis with periappendiceal abscess. ${ }^{5}$ In our case, however, the patient's presentation and pain complaints were more indolent than acute in nature. Her initial examination and benign radiologic findings, coupled with her young age, persistent localized pain complaints, and medical history of PID, led to the diagnosis of chronic pelvic pain. It is well known that women who acquire acute PID are at risk for long- term sequelae including recurrent PID and chronic pelvic pain. ${ }^{8,9}$

Chronic pelvic pain can be caused by a variety of conditions. The possible sources encompass at least 5 major physiological systems: gastrointestinal, urologic, gynecologic or reproductive, musculoskeletal, and neurological/ psychological. Pinpointing the true etiology can be daunting, and the etiology is often multifactorial. Our patient was treated with various formulations and combinations of antibiotics, nonsteroidal anti-inflammatory agents, estrogen-progestin contraceptives, and gonadotropin-releasing hormone agonists over the course of 20 months without any resolution of her symptoms. In these difficult cases it is imperative to remember that some women will have $>1$ condition contributing to their pain, and although it is low in the differential diagnosis of a 32-yearold woman, malignancy must be considered. We propose that when medical therapy for chronic pelvic pain fails a 3- to 6-month empiric trial, regardless of the suspected etiology, the patient should undergo a full re-evaluation. This includes an analysis of the history to date and review of the current laboratory testing results and imaging, followed by re-examination, reimaging, appropriate referrals when indicated, and diagnostic laparoscopy.

In these cases laparoscopic visualization with histologic examination is the definitive diagnostic modality. The literature has consistently shown that the most reliable method of detection of malignancy is the combination of laparoscopic visualization and frozen-section analysis. ${ }^{10,11}$ Moreover, primary mucinous appendiceal adenocarcinoma metastatic to the ovaries may mimic advanced-stage ovarian mucinous carcinoma. ${ }^{12}$ Because the surgical and chemotherapeutic management of these two distinct malignancies differs greatly, an accurate histologic diagnosis at the time of surgery is crucial to the initiation of appropriate therapy. The fundamental diagnostic combination of laparoscopic visualization and frozen-section analysis ultimately led to our patient's diagnosis and treatment. In this case, continued medical management alone for her chronic pelvic pain, without a full re-evaluation, including at least repeat imaging and, more importantly, diagnostic laparoscopy, would have carried a much higher mortality risk.

\section{CONCLUSION}

Chronic pelvic pain is a frequently encountered clinical condition, whereas primary appendiceal adenocarcinoma is exceptionally rare. The initial evaluation of patients with 
chronic pelvic pain must be thorough and should include a complete history, physical examination, and appropriate imaging and laboratory studies. We propose that when medical therapy for presumed chronic pelvic pain fails a 3- to 6-month empiric trial, the patient should undergo a full re-evaluation including diagnostic laparoscopy. Even in the young female patient, the possibility of malignancy must always be considered within the differential diagnosis of chronic pelvic pain. It is important for the gynecologist and gynecologic oncologist to be familiar with the clinical and pathologic features in addition to the surgical management of these malignancies because primary appendiceal adenocarcinomas may masquerade as chronic pelvic pain.

\section{References:}

1. Ozakyol AH, Saricam T, Kabukcuoglu S, Caga T, Erenoglu E. Primary appendiceal adenocarcinoma. Am J Clin Oncol. 1999; 22:458-459.

2. McCusker ME, Coté TR, Clegg LX, Sobin LH. Primary malignant neoplasms of the appendix: a population-based study from the Surveillance, Epidemiology and End Results program, 19731998. Cancer. 2002;94:3307-3312.

3. Ito H, Osteen RT, Bleday R, et al. Appendiceal adenocarcinoma: long-term outcomes after surgical therapy. Dis Colon Rectum. 2004;47:474-480.

4. Bucher P, Mathe Z, Demirag A, Morel P. Appendix tumors in the era of laparoscopic appendectomy. Surg Endosc. 2004;18: 1063-1066.
5. Sugarbaker PH, Alderman R, Edwards G, et al. Prospective morbidity and mortality assessment of cytoreductive surgery plus perioperative intraperitoneal chemotherapy to treat peritoneal dissemination of appendiceal mucinous malignancy. Ann Surg Oncol. 2006;13:635-644.

6. Ronnett BM, Shmookler BM, Diener-West M, Sugarbaker $\mathrm{PH}$, Kurman RJ. Immunohistochemical evidence supporting the appendiceal origin of pseudomyxoma peritonei in women. Int J Gynecol Pathol. 1997;16(1):1-9.

7. Oya S, Miyata K, Yuasa N, et al. Early carcinoma of the appendix vermiformis. Dig Endosc. 2009;21:53-55.

8. Haggerty CL, Schulz R, Ness RB. Lower quality of life among women with chronic pelvic pain after pelvic inflammatory disease. Obstet Gynecol. 2003;102(5):934-939.

9. Thurman AR, Soper DE. Sequelae. In: Sweet RL, Wiesenfeld HC, eds. Pelvic Inflammatory Disease. London, England: Taylor \& Francis; 2006:69-84.

10. Nezhat F, Nezhat C, Welander CE, Benigno B. Four ovarian cancers diagnosed during laparoscopic management of 1011 women with adnexal masses. Am J Obstet Gynecol. 1992;167:790-796.

11. Lang JH, Zhang JJ, Feng FZ, et al. Role of laparoscopy in the diagnosis and treatment of adnexal masses. Chin Med J (Engl) 2006;119(3):202-206.

12. Young RH, Gilks CB, Scully RE. Mucinous tumors of the appendix associated with mucinous tumors of the ovary and pseudomyxoma peritonei. A clinicopathological analysis of 22 cases supporting an origin in the appendix. Am J Surg Pathol. 1991;15(5):415-429. 